\title{
A Dynamic Approach to Identification of Multiple Harmonic Sources in Power Distribution Systems
}

\author{
Awajiokiche Ujile, Zhengtao Ding \\ School of Electrical and Electronic Engineering, University of Manchester, M13 9PL, United Kingdom
}

\begin{abstract}
This paper provides a novel algorithm for identifying harmonic sources in power distribution systems. This algorithm is developed based on an observer design to carry out harmonic estimation for a combination of suspicious nodes. The estimation error is analysed to determine the existence of harmonic sources in the specified node combinations. This approach is used to determine the source of both single and multiple harmonic sources in distribution systems with time varying load parameters. For systems with a large number of suspicious nodes, the system may be divided into sub-systems and the algorithm is applied to each sub-system to identify the harmonic sources present. Simulations are carried out on a benchmark IEEE distribution test feeder for both single and multiple harmonic sources and a number of scenarios are simulated to verify the accuracy and robustness of the proposed approach. The results show that the node combinations which represent the harmonic sources yield an estimation error which approaches zero asymptotically.
\end{abstract}

Keywords: Harmonics, Observer, Power distribution systems

\section{Introduction}

The proliferation of power distribution systems with harmonic disturbances is as a result of the increasing use of power electronic devices in the design of household and industrial equipment. IEEE Std 519-1992 [1] provides specifications for the allowable harmonic content in power systems. To comply with these standards, utility companies need to be aware of the exact source of harmonic injections in the system at any given time. This information may be instrumental in the design of an appropriate mechanism to extract or nullify the effects of the harmonic injections or penalising those responsible.

A number of methods have been reported in literature for determining the source of harmonic injections in power systems. In general, two major approaches to harmonic source identification are proposed: single

Email addresses: awaiujile@gmail.com (Awajiokiche Ujile), zhengtao.ding@manchester.ac.uk (Zhengtao Ding) 
point methods and multiple point methods. In single point methods, the source of harmonic injections are determined by taking measurements at a single point in the system, usually the point of common coupling (PCC), and the harmonic source is determined to either be upstream or downstream from that point. This method apportions the responsibility of harmonic injection either to the customer or the utility. Some techniques based on single point methods include critical impedance $[2,3,4,5]$, neural networks $[6]$ and nonactive power [7]. Another single point method, proposed in [8], utilizes a Norton equivalent circuit to represent a model of the power system comprising of the customer side and the utility side. A general review of various single point methods of harmonic source identification was carried out in [9]. As much as the single point method is effective in providing a general overview of the source of harmonic disturbances, it does not give accurate information about the exact node where the harmonic injections occur. For systems with multiple harmonic sources, the harmonic distortion at the PCC is usually the result of an aggregate of the multiple distortions coming from various parts of the system. A major concern is that the multiple harmonic sources may offset each other due to the phase angle differences and the time varying nature of the loads. Hence, the distortion at the PCC may not accurately represent the harmonic injections emanating from the various nodes in the system. Therefore, the multiple point approach to harmonic source identification is preferred to the single-point method due to its ability to identify the exact nodes where the harmonic injections occur as well as the intensity of the harmonic injections.

The most common method of multiple-point harmonic source identification is harmonic state estimation (HSE). HSE involves the estimation of the states of the system using available measurements. The states may be chosen to be the voltage magnitudes and phase angles at all nodes in the system or the current injections, depending on the method used to carry out HSE. Two approaches to HSE are available in literature: static HSE $[10,11]$ and dynamic HSE[12, 13, 14]. With dynamic HSE, a dynamic representation of the time evolution of the system is used for state estimation. Utility companies are thereby able to detect rapid changes in the system and respond accordingly. In addition dynamic HSE gives a more accurate representation of state estimation when there are load changes compared to static HSE. The most common method of dynamic HSE is the Kalman filter $[15,16]$ and its modifications such as adaptive Kalman filter [13] and robust extended Kalman filter [14]. The adaptive Kalman filter proposed in [13] uses two process noise covariant, $Q$, models to carry out harmonic estimation and this eliminates the requirement for the calculation of an optimal $Q$ model. A time domain approach to HSE was proposed in $[17,18]$. This method applies the numerical differentiation approach to determine a fast periodic steady-state solution in the time domain and the the Kalman filter is used to solve the HSE problem. The Kalman filter HSE methods are 
applied for estimation of the harmonic node voltages in a given system. However, they have not been applied to distribution system harmonic source identification. Also, the time domain approach proposed in [17] was not extended to harmonic source identification.

Apart from HSE, other methods have been previously applied to identification of harmonic sources. In [19], the theory of statistical inference is used to determine the harmonic index of a particular load in the system. The direction of harmonic power flow is applied in [20] to rank the nodes in the system to suspicious and non-suspicious nodes. Binary particle swarm optimization is then used to determine the optimum locations to place measurement devices. One drawback of this approach used is that an assumption that harmonic voltage phasor measurements are available for all the nodes in the system is applied. However, it is not cost effective to obtain measurements at all nodes in a given system, hence the need for optimal measurement placement methods and observability analysis. In [21], a Bayesian approach was applied to harmonic source estimation. A dynamic representation of the system was presented and the forcing term (which represents the harmonic current injections) was determined from measured and pseudomeasured data. A cascade correlation system is also presented in [22] for determining multiple harmonic sources in a given system.

In this paper an algorithm is developed to identify multiple harmonic sources in power distribution systems. This algorithm utilizes observer design to estimate the system states. The fundamental frequency is assumed to be known and harmonics are estimated from available measurements. For ideal scenarios, the number of harmonic sources in the system is unknown. The nodes which contain nonlinear loads are determined by using a combinational approach and the estimation errors are analysed for each combination. Systems with a large number of suspicious nodes are divided into subsystems to improve computational efficiency. The relationship between node current injections and the state variables is then formulated. This is used to calculate the actual current injections at each node. Determination of the exact magnitude and phase of the injected harmonic current may be achieved by using the iterative observer algorithm proposed in [23]. Observers have been widely used to estimate disturbances in various industrial systems [24, 25]. The observer based method proposed in this paper accurately determines the source of harmonic injections in a distribution system using a time-domain dynamic model. This time-domain approach is advantageous due to its ability to adapt to the dynamic nature of distribution system loads with time. This method is also able to determine the harmonic sources irrespective of the rapid changes in the harmonic source in the system. In addition, the algorithm presented is able to determine the presence of multiple harmonic sources in real time as well as adapt to rapid changes in the nature of the harmonic sources with time. The 
observer-based algorithm proposed in this paper takes into account the time varying nature of distribution system loads and harmonic sources. The Kalman filter requires knowledge of the process and measurement noise covariance parameters. This makes the proposed algorithm more straightforward because knowledge of these parameters is not required for implementation. Simulations are included to verify the accuracy of the proposed method.

\section{Problem formulation}

Consider a power distribution system represented in state-space form as

$$
\begin{aligned}
& \dot{x}=A x+B u+E w \\
& y=C x+D u
\end{aligned}
$$

where $x \in \mathbb{R}^{n}$ is the state vector, $y$ represents the measurements, $u$ is the system input, $A, B, C, D$ are known matrices, $E$ represents the disturbance matrix and $w$ is the harmonic disturbance given by

$$
w(t)=\sum_{h=2}^{f} H_{h} \sin \left(\omega_{h} t+\phi_{h}\right)
$$

where for $h=2 \ldots f, H_{h}, \phi_{h} \in \mathbb{R}$ represent the magnitude and phase angles respectively, $\omega_{h}=2 \pi f h, f$ is the fundamental frequency and $h$ is the harmonic order.

In practical systems, $E$ is unknown. This is because the exact states in the system where the harmonic injections occur are unknown.

Assumption 1. The harmonic source is not directly measured. As a result, the state space representation in (1) and (2) does not indicate any harmonic injection in the outputs.

Remark 1. The existence of a current measurement at the direct source of harmonic injections in the system creates another term in the measurement equation. The coefficient of this harmonic disturbance in the measurement is unknown.

The system state-space matrices are determined using the methods proposed in [26] and [17]. The states of the distribution system are node voltages and line currents.

The aim of this work is to determine the harmonic sources in a given power distribution system using an observer-based algorithm. A time domain dynamic approach is presented in this paper where the system is modelled as a process at the fundamental frequency and the harmonic injections are taken as disturbances to the system. This time domain representation is effective where the power system has time-varying load and harmonic source parameters. 
Power distribution systems are subjected to a wide range of loads, both linear and nonlinear. The assumptions in [27] are applied in this paper for modelling the distribution system parameters. Linear loads may be represented as constant RL impedance while nonlinear loads are modelled as a linear load in parallel with a harmonic current generator [20,28].

\section{Observer based harmonic source identification}

An observer-based approach is proposed in this section for determining the harmonic sources in a given power system. The state observer utilizes the state space equations given in (1) and (2) for harmonic source identification. The dynamics of the harmonic disturbance, $w$, may be described as a linear exosystem:

$$
\begin{aligned}
& \dot{w}=S w \\
& \mu=g^{T} w
\end{aligned}
$$

where $S=\operatorname{diag}\left(S_{2}, S_{3} \ldots S_{h}\right), S_{h}=\left[\begin{array}{cc}0 & \omega_{h} \\ -\omega_{h} & 0\end{array}\right], \omega_{h}=2 \pi f h, w=\left[w_{2}, w_{3} \ldots w_{h}\right]$ and $\mu=\left[\mu_{1}, \mu_{2} \ldots \mu_{h}\right]^{T}, h$ is a set of integers $\mathcal{H}=\left\{h_{l}\right\}$ for $l=2 \ldots f . l=1$, which is the fundamental frequency is not considered to be a disturbance in the system. The augmented form of the dynamic equation in (1), (2) becomes:

$$
\left[\begin{array}{c}
\dot{x} \\
\dot{w}
\end{array}\right]=\left[\begin{array}{ll}
A & E \\
0 & S
\end{array}\right]\left[\begin{array}{l}
x \\
w
\end{array}\right]+\left[\begin{array}{l}
B \\
0
\end{array}\right] u
$$

Applying the Luenberger observer to the augmented system (6) yields the state observer:

$$
\left[\begin{array}{c}
\dot{\hat{x}} \\
\dot{\hat{w}}
\end{array}\right]=\left[\begin{array}{cc}
A & E \\
0 & S
\end{array}\right]\left[\begin{array}{l}
\hat{x} \\
\hat{w}
\end{array}\right]+\left[\begin{array}{l}
B \\
0
\end{array}\right] u+\left[\begin{array}{l}
L \\
\tilde{L}
\end{array}\right](y-C \hat{x}-D u)
$$

The observer given in (7) provides estimates of all the states in the system. These augmented states consist of the node voltages, line currents, and the harmonic disturbances.

To obtain accurate state estimates using the augmented observer given in (7), an accurate representation of $E$ is required. In practical systems, the disturbance matrix, $E$, is unknown. Hence, an observer-based approach is applied to the observer design to determine $E$. If $S \in \mathbb{R}^{q}$ and $A \in \mathbb{R}^{n}$, then $E \in \mathbb{R}^{n \times q}$. Assuming the harmonic disturbance enters the system through the $k^{t h}$ state. The $k^{\text {th }}$ row of the disturbance matrix 
becomes

$$
E_{k}=\left[\begin{array}{ll}
e_{i} & e_{i} \ldots e_{i}
\end{array}\right]
$$

where $k$ is the state where the disturbance is injected in the system, $e_{i}$ is the harmonic injection index at node $i$.

Definition 1. The harmonic injection index, $e_{i}$, at a given node, $i$, is the coefficient of the harmonic disturbance injected into the system states at that particular node.

This index constitutes the elements of the matrix, $E$ and is calculated from the shunt admittance of all lines adjacent to the specified node. The shunt admittance is given by

$$
Y=G+j B_{s}
$$

where $G$ is the conductance and $B_{s}$ is the susceptance. The shunt capacitance, $C_{s}$, is calculated using (10).

$$
B_{s}=2 \pi f C_{s}
$$

where $f$ is the fundamental frequency and $B_{s}$ is the capacitive susceptance. The harmonic injection index at a given node is calculated as twice the inverse of the sum of shunt capacitances at all the branches adjacent to that node. For a $p$ node system, the harmonic injection index at node $i$ is computed as:

$$
e_{i}=\frac{2}{\sum_{r=1}^{p} C_{i r}}
$$

where $C_{i r}$ is the capacitance for line $i-r$, which is calculated from (10).

The system nodes are partitioned into suspicious and non-suspicious nodes. Suspicious nodes are nodes which are connected to loads and non-suspicious nodes have no loads, hence there is no chance of them being harmonic sources. To obtain an accurate representation of the harmonic disturbance matrix, $E$, the row of $E$ which corresponds to the suspicious node voltage is replaced with (8) for each suspicious node. State estimation is then carried out using (7). If the estimation error approaches zero asymptotically, then $E$ is taken to be accurate and the suspicious node $i$ is the harmonic source.

For a $p$ node system, Kirchhoff's current law is used to determine the current injection matrix at node $i$ as:

$$
I_{i}=\sum_{j=1}^{p} I_{i j}+\sum I_{L, i}
$$


where $I_{i j}$ is the current at branch $i-j$ and $I_{L, i}$ represents the load current at node $i$.

The current injection matrix is used to compute the harmonic current injections from the estimated state variables. The direction of current flow is taken into account when computing the current injections at the nodes. A relationship between the node current injections and the state estimates is established as:

$$
I_{i}=M \bar{x}
$$

where $\bar{x}=\left[\begin{array}{ll}x & w\end{array}\right]^{T}$ is the augmented state variable and $M \in \mathbb{R}^{p \times n}$ is computed as:

$$
M(a, b)= \begin{cases}1 & \text { If current flows from node } a \text { to the } \\ & \text { receiving end of the } b^{t h} \text { state } \\ & \text { OR if the load current at the } b^{\text {th }} \\ & \text { state is connected to node } a \\ -1 & \text { If current flows from the sending } \\ & \text { end of the } b^{\text {th }} \text { state to node } a \\ 0 \quad & \text { Otherwise }\end{cases}
$$

where $p$ represents the number of nodes in the given system and $n$ is the number of states.

\section{Identification of multiple harmonic sources}

In power distribution systems, determining the exact number of harmonic sources at any given time may be challenging due to the rapidly changing loads. A combination approach is therefore presented for determining the source of harmonic injections in the system using a time domain dynamic model. Nodes with no load are eliminated from the algorithm to improve computation time and reduce redundancy. For a $p$ node system, with $s$ loads, there are a maximum of $s$ simultaneous harmonic sources. Accurate identification of the disturbance matrix, $E$ is crucial in determining multiple harmonic sources in the system. The dimensions of this matrix varies depending on the number of harmonic sources present. For instance, if there are $t$ harmonic sources in a $p$ node system with $s$ loads, then $E \in \mathbb{R}^{n \times t q}$, where $n$ represents the number of states and $q$ represents the dimensions of $S$. As $E$ changes with the number of harmonic sources, $S \in \mathbb{R}^{t q \times t q}$ populates the diagonal of the matrix, $S$. The algorithm for harmonic source identification in a 
given power distribution system is as follows:

1: Determine the state space matrices for the system using the method proposed in the appendix of [17].

2: Partition the system nodes into suspicious and non-suspicious nodes.

3: Calculate the harmonic injection index, $e_{s}$, for each suspicious node $s$ using (11)

4: Select the states which correspond to node voltages for suspicious nodes.

5: For each suspicious node, $s$, create $E_{s}$ by populating the row of $E$ which represents each state selected in step 4 with the harmonic injection index at that node.

$$
E_{s}=\left[\begin{array}{cccc}
0 & 0 & \ldots & 0 \\
\vdots & \vdots & \ddots & \vdots \\
e_{s} & e_{s} & \ldots & e_{s} \\
0 & 0 & \ldots & 0
\end{array}\right]
$$

6: for $t=1$ to $s, t$ represents the number of simultaneous harmonic sources and $s$ is the number of suspicious nodes do

7: Determine the combinations for harmonic sources, $d=\left(\begin{array}{l}s \\ t\end{array}\right)$

8: $\quad$ for $v=1$ to $z, z$ is the number of combinations for $t$ harmonic sources, do

9: $\quad$ Create $E_{t} \in \mathbb{R}^{n \times s t}$, which is a matrix with diagonal consisting of $E$ for all possible harmonic source combinations.

10: $\quad$ Create $S \in \mathbb{R}^{q t \times q t}$ as $\operatorname{diag}([S \ldots S])$

11: $\quad$ Design a state observer given by

$$
\begin{aligned}
{\left[\begin{array}{c}
\dot{\hat{x}} \\
\dot{\hat{w}}
\end{array}\right] } & =\left[\begin{array}{rr}
A & E_{t} \\
0 & S
\end{array}\right]\left[\begin{array}{l}
\hat{x} \\
\hat{w}
\end{array}\right]+\left[\begin{array}{l}
B \\
0
\end{array}\right] u+L(y \\
& -C \hat{x}-D u)
\end{aligned}
$$

12: $\quad$ Determine the current injection matrix using (14).

where $L$ is designed such that $\bar{A}-L \bar{C}$ is Hurwitz [29], $\bar{A}=\left[\begin{array}{cc}A & E_{t} \\ 0 & S\end{array}\right]$ and $\bar{C}=\left[\begin{array}{ll}C & 0\end{array}\right]$

13: $\quad$ if $r_{t}=y-C \hat{x}-D u$ and $\lim _{t \rightarrow \infty} r_{t}=0$ then

14: $\quad$ There are $t$ harmonic sources in the system located at the nodes in the combination, $d(v)$. 


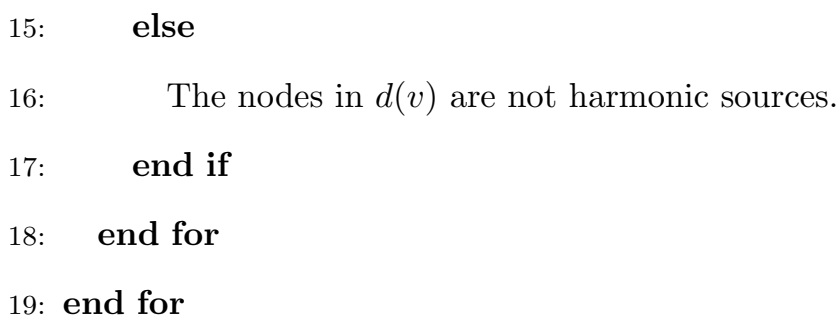

Remark 2. The condition that verifies the number and locations of harmonic sources which is determined by examining the estimation error applies to all measurements taken throughout the system.

For large systems with a large number of suspicious nodes, the system can be split into sub-systems and the observer-based algorithm is applied to each sub-system to determine the harmonic source. This reduces the number of combinations, thereby improving computation time and cost. The block diagram for application of the observer to harmonic source identification is shown in Fig. 1.

\section{Case study}

The validity of the proposed harmonic source estimator is verified by carrying out simulations on the IEEE 13-node test feeder, given in [30, 27]. A one-line diagram of the system is shown in Fig. 2 . A voltage source behind an impedance is connected to node 50, with short circuit capacity given by 1100MVA, transformers are represented by a two-winding model and lines are modelled using $\pi$ branches with length dependent parameters. In distribution systems, the shunt admittance is negligible due to the short length of the lines. The line parameters for this system are obtained from [27].

Linear loads are modelled as RL impedance with data given in Table 1, while nonlinear loads are modelled as a linear load in parallel with a harmonic current generator. The system states include node voltages, line currents and load currents. Ten synchronized measurements are taken throughout the system. These measurements consist of node voltages and line currents and they include: $V_{31}, V_{33}, V_{45}, V_{71}, V_{92}, V_{84}, I_{31-32}$, $I_{32-45}, I_{71-92}, I_{71-84}$

From Table 1, we can deduce that nodes 45, 46, 71, 75, 92, 52 and 911 are the suspicious nodes because they have loads connected to them. All other nodes in the distribution system are non-suspicious nodes, hence they are not included in the harmonic source identification process. Three types of harmonic loads are simulated in this study: fluorescent lamps, adjustable speed drives and other composite residential loads. A number of scenarios are simulated to verify the validity of the proposed method 


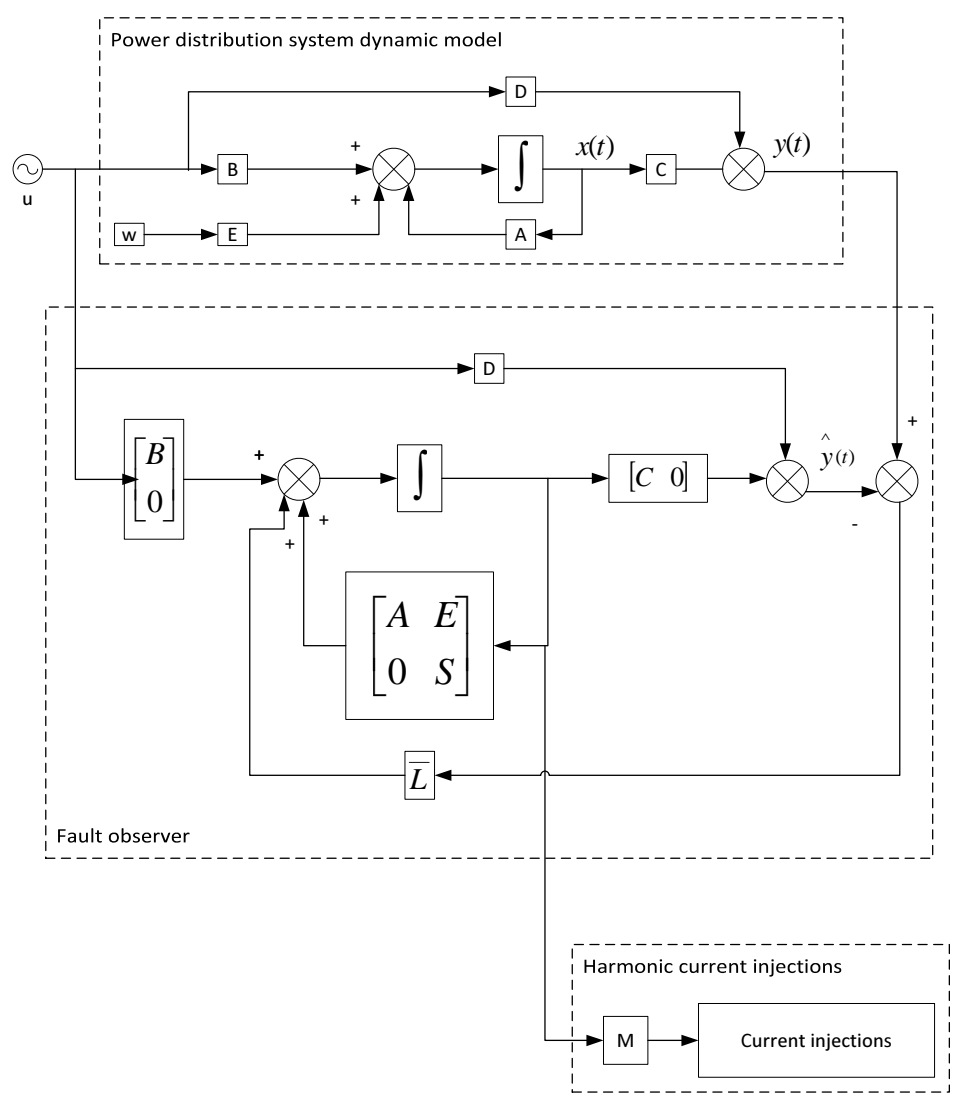

Figure 1: Observer based harmonic source identification

\subsection{Single harmonic source}

Consider the system configuration where there is a single nonlinear load at node 71 with harmonic spectra shown in Table 2. The performance of the harmonic source estimator is evaluated by analysing the mean squared error (MSE) of all the estimates. The MSE is expressed as

$$
M S E=\frac{1}{s} \sum_{m=1}^{s}\left|y_{m}-\hat{y}_{m}\right|^{2}
$$

A threshold is set at 0.05 for determining the harmonic source based on the MSE of the estimation errors. If the MSE is below the threshold, this is an indication that the estimation errors approach zero asymptotically and the corresponding node combination is taken to be the harmonic source. However, if the MSE is above the threshold, then the node combination is not the harmonic source.

The data presented in table 3 shows the MSE of the steady-state estimation errors computed for all measurements for all combinations when there is a single harmonic source. From the table, it is apparent 


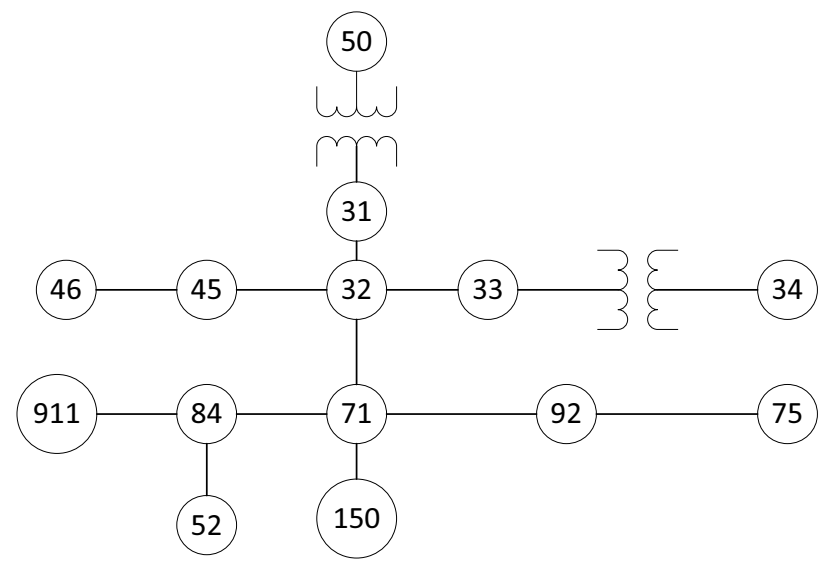

Figure 2: One line diagram of the IEEE 13-node feeder

Table 1: Distribution system load data

\begin{tabular}{c|c|c}
\hline Node & $P(\mathrm{~kW})$ & $Q(\mathrm{kVar})$ \\
\hline 45 & 170.53 & 125.09 \\
\hline 46 & 230.22 & 131.97 \\
\hline 71 & 383.70 & 219.95 \\
\hline 75 & 486.02 & 189.07 \\
\hline 92 & 170.53 & 151.38 \\
\hline 52 & 127.90 & 85.79 \\
\hline 911 & 170.53 & 80.74 \\
\hline
\end{tabular}

that node 71 is the only instance where the MSE for all measurements is below the 0.05 threshold. Hence node 71 is identified as the harmonic source.

Subsequent to identifying node 71 as the harmonic source, the current injection matrix, which is determined using the expression in (12) is used to determine the magnitude of the harmonic current injection at node 71. Fig. 3 shows the harmonic current injection at node 71.

\subsection{Time varying harmonic injections}

The time varying nature of harmonic loads in distribution systems is a factor to be considered when determining harmonic sources. The method of harmonic source identification proposed in this paper is capable of adapting to the rapidly changing load profiles of power distribution systems. Two scenarios are simulated in this section: the first is the sudden harmonic injection in the system and the second is a rapid change in harmonic sources. 
Table 2: Harmonic spectrum for node 71

\begin{tabular}{c|c|c}
\hline Harmonic & Magnitude $(\%)$ & Phase $\left(^{\circ}\right)$ \\
\hline 1 & 10000 & -31.18 \\
\hline 3 & 6.42 & 243.80 \\
\hline 5 & 3.33 & -260.70 \\
\hline 7 & 0.63 & 178.90 \\
\hline
\end{tabular}

Table 3: Mean squared error in all system measurements for single harmonic source

\begin{tabular}{c|c|c|c|c|c|c|c|c|c|c}
\hline \multicolumn{1}{c}{ Node } & $V_{31}$ & $V_{33}$ & $V_{92}$ & $V_{71}$ & $V_{84}$ & $V_{45}$ & $I_{31-32}$ & $I_{32-45}$ & $I_{71-84}$ & $I_{71-92}$ \\
\hline 45 & 0.02 & 0.91 & 0.15 & 0.16 & 0.17 & 2.59 & 0.51 & 3.03 & 0.01 & 1.16 \\
\hline 46 & 0.02 & 0.92 & 0.15 & 0.16 & 0.17 & 2.62 & 0.52 & 3.02 & 0.01 & 1.15 \\
\hline 71 & $\mathbf{0 . 0 2}$ & $\mathbf{0 . 0 3}$ & $\mathbf{0 . 0 3}$ & $\mathbf{0 . 0 3}$ & $\mathbf{0 . 0 4}$ & $\mathbf{0 . 0 2}$ & $\mathbf{0 . 0 1}$ & $\mathbf{0 . 0 0}$ & $\mathbf{0 . 0 0}$ & $\mathbf{0 . 0 3}$ \\
\hline 92 & 0.02 & 0.03 & 0.54 & 0.06 & 0.06 & 0.02 & 0.01 & 0.00 & 0.01 & 0.04 \\
\hline 75 & 0.02 & 0.03 & 0.54 & 0.06 & 0.06 & 0.02 & 0.01 & 0.00 & 0.01 & 0.04 \\
\hline 911 & 0.02 & 0.07 & 0.22 & 0.24 & 0.03 & 0.06 & 0.04 & 0.00 & 0.31 & 1.15 \\
\hline 52 & 0.02 & 0.08 & 0.22 & 0.24 & 0.03 & 0.07 & 0.04 & 0.00 & 0.31 & 1.14 \\
\hline
\end{tabular}

\subsubsection{Sudden harmonic injection}

Suppose the magnitudes of the harmonic currents at node 71 are time varying as shown in (18), (19) and (20).

$$
\begin{aligned}
& H_{3}= \begin{cases}0 & t<0.5 s \\
6.42 \% & t>0.5 s\end{cases} \\
& H_{5}= \begin{cases}0 & t<0.5 s \\
3.33 \% & t>0.5 s\end{cases} \\
& H_{7}= \begin{cases}0 & t<0.5 s \\
0.63 \% & t>0.5 s\end{cases}
\end{aligned}
$$

The MSE is computed for the steady state estimation error for all measurements. Fig. 4 shows the MSE for all 7 combinations for a single harmonic source with time varying harmonic injections. Logarithmic scales are used to show the MSE for ease of readability, due to the variations between values of the MSE. Although some MSE values appear close to the threshold, they are clearly larger in value than the threshold and only appear close due to the logarithmic scale used to present the results. The tick in the figure shows the MSE 


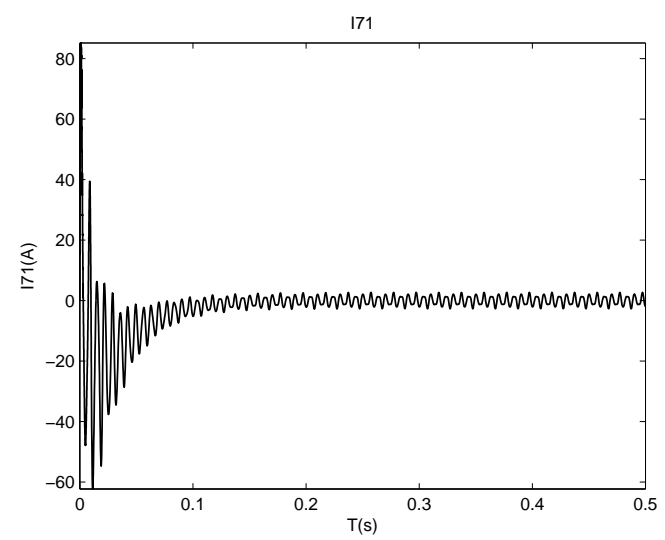

Figure 3: Harmonic current injection at node 71 for single harmonic source

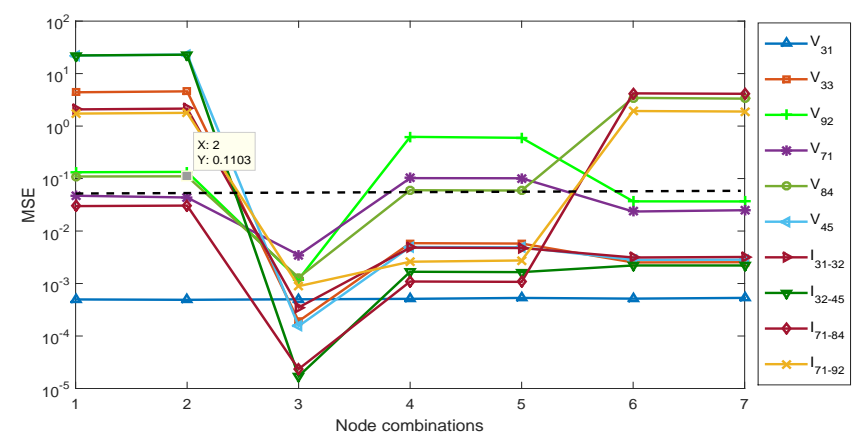

Figure 4: Mean squared error with time varying harmonic injections

for $V_{84}$ at combination 2 as 0.1103 , which is clearly larger than the threshold.

To accurately identify the harmonic source, the MSE must be below the threshold for all measurements. From the figure, it is apparent that the third combination is the only instance where the MSE for all measurements are below the threshold. Therefore, the third combination, which represents node 71 , is identified as the harmonic source. Figure 5 shows the harmonic current injections at node 71 . The figure shows that at node 71, after an initial transient, there is no harmonic injection when $t<0.5 s$. When $t>0.5 \mathrm{~s}$, the harmonic current injection is shown in the figure. This shows that the proposed algorithm is able to adapt to variations in harmonic injections in the system.

\subsubsection{Sudden change in harmonic source}

Suppose at a certain time instant, $t$, the harmonic source changes from one node to another. If at $t<0.5 \mathrm{~s}$, the harmonic source is at node 71 and at $t>0.5 \mathrm{~s}$, the harmonic source at node 71 is switched off and the one at node 75 is switched on. The harmonic spectra used for both cases are shown in tables 2 


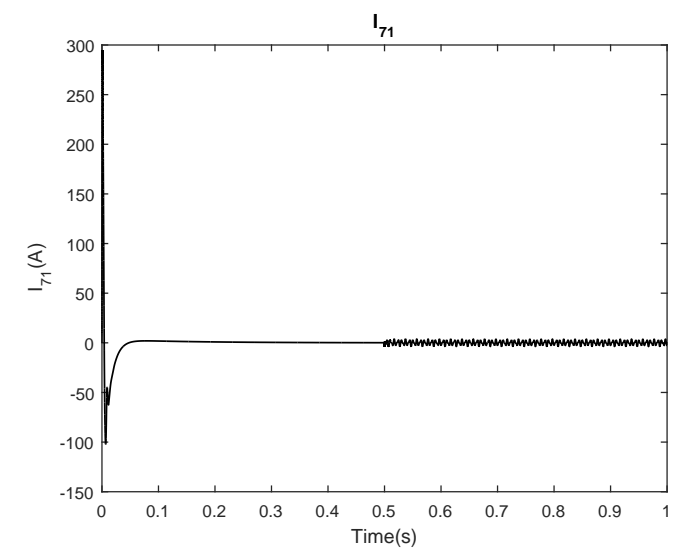

Figure 5: Harmonic current injections at node 71 with sudden harmonic injection at $0.5 \mathrm{~s}$

and 4. Fig. 6 and 7 show the MSE for the node combinations 71 and 75 respectively, where the MSE at $0.5 \mathrm{~s}$ represents transients. From Fig. 6 , it is clear that at $t<0.5 \mathrm{~s}$, node 71 is accurately identified as the harmonic source, as the MSE for all measurements is below the 0.05 threshold. However, at $t>0.5 \mathrm{~s}$, node 71 ceases to be the harmonic source and after an initial transient, the maximum MSE for the measurements settles at 0.7744 , which is above the threshold. Fig. 7 shows that the steady-state MSE at $t>0.5 s$ is 0.0058 , which is below the threshold, hence identifying node 75 as the harmonic source. This verifies the capability of the proposed method of harmonic source identification to adapt to rapid changes in harmonic sources within the power distribution system.

\subsection{Multiple harmonic sources with noisy measurements}

The possibility of having a single harmonic source in any power distribution system is unlikely due to the numerous customers who may simultaneously use nonlinear loads. There is no prior knowledge of the locations of these nonlinear loads. Hence, it is important to determine the exact node where these multiple loads exist. For the case study presented, assuming two harmonic sources are present simultaneously at node 71 and 45 with spectra given in Tables 2 and 5 respectively. Random noise with Gaussian distribution is also added to the measurements. Due to the size of the system and the number of suspicious nodes, there are 127 possible combinations. To reduce the computational burden, this system is divided into two sub-systems and the algorithm is applied on both sub-systems to determine the harmonic source. Fig. 8 shows both sub-systems. The first sub-system contains the measurements $V_{31}, V_{33}, V_{45}, I_{31-32}$ and $I_{32-45}$, while the second sub-system contains the measurements $V_{71}, V_{92}, V_{84}, I_{71-92}$ and $I_{71-84}$. For the first sub-system, the suspicious nodes are 45 and 46 , therefore the number of combinations reduces to 3 . The suspicious nodes for the second sub-system are 71, 92, 75, 911 and 52, therefore there are 31 combinations 
Table 4: Harmonic spectra for node 75

\begin{tabular}{c|c|c}
\hline Harmonic & Magnitude $(\%)$ & Phase $\left({ }^{\circ}\right)$ \\
\hline 1 & 100.00 & -68.72 \\
\hline 3 & 3.58 & 245.88 \\
\hline 5 & 1.25 & 311.48 \\
\hline 7 & 0.43 & 110.18 \\
\hline
\end{tabular}

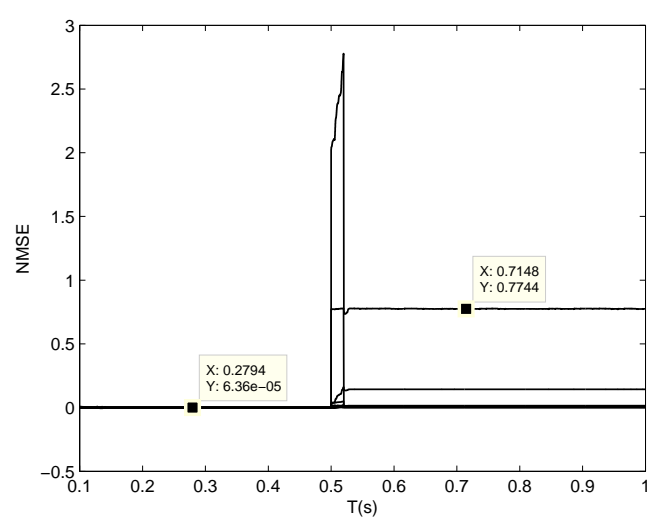

Figure 6: MSE for node combination 71 with change in harmonic source at $0.5 \mathrm{~s}$

for the second sub-system. The total number of combinations has therefore been reduced to 34, compared to 127 combinations for the entire system.

The threshold of the MSE is set at 0.05. Results shown in Table 6 depict the MSE for the first subsystem. From the table, it is apparent that the node combination 45 is the only instance where the MSE is below the set threshold. Hence this node is identified as the harmonic source for this sub-system.

Considering the second sub-system, using a threshold of 0.05 , the algorithm is applied to identify the harmonic sources. Fig. 9 shows the MSE for all 31 combinations. The figure shows that combination 1 is the only instance where the MSE is below the 0.05 threshold. In this case combination 1 corresponds to node 71 . Therefore, node 71 is identified as the harmonic source.

\subsection{Additional harmonic source}

Suppose after identifying these harmonic sources, an additional harmonic source is introduced at node 75 with spectra shown in Table 4. From previous multiple harmonic source identification, the sub-system where the additional harmonic source is introduced provides an estimation error which ceases to approach zero asymptotically. The source of the additional harmonic injection is determined by only applying the proposed algorithm on the sub-system affected. The nodes which have been previously identified as harmonic sources 


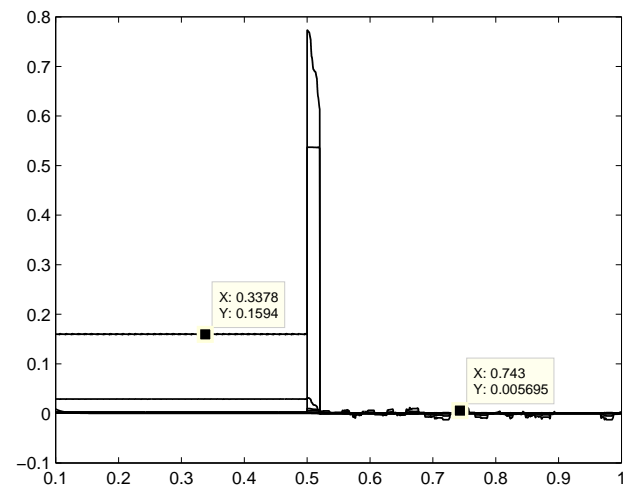

Figure 7: MSE for node combination 75 with change in harmonic source at $0.5 \mathrm{~s}$

Table 5: Harmonic spectra for node 45

\begin{tabular}{c|c|c}
\hline Harmonic & Magnitude $(\%)$ & $\left.\operatorname{Phase}^{\circ}\right)$ \\
\hline 1 & 100.00 & -37.29 \\
\hline 3 & 14.98 & 245.88 \\
\hline 5 & 5.22 & 311.48 \\
\hline 7 & 1.80 & 110.18 \\
\hline
\end{tabular}

are removed from the list of suspicious nodes, thereby improving computation time.

For the second sub-system, the previously identified harmonic source is node 71 . Hence, the new set of suspicious nodes is $\{92,75,911,52\}$. Applying the observer-based algorithm gives the results shown in Fig. 10. The figure shows that combinations 2 has the MSE below the threshold of 0.5 for all measurements. These combinations represent node 75. All other combinations have the MSE above the threshold. Hence, node 75 is identified as the additional harmonic source.

\section{Conclusion}

This paper has presented an observer based approach to harmonic source identification in power distribution systems. A combination of suspicious nodes is used to ascertain the harmonic sources by analysing the mean squared error for the combinations. Simulation results show that the proposed method is capable of determining the sources of harmonic injections in the presence of measurement noise or variations in the magnitude or location of the harmonic source. Also, additional harmonic sources may be determined subsequent to identifying previous sources. Large systems can be divided into smaller sub-systems to improve the computational efficiency. 


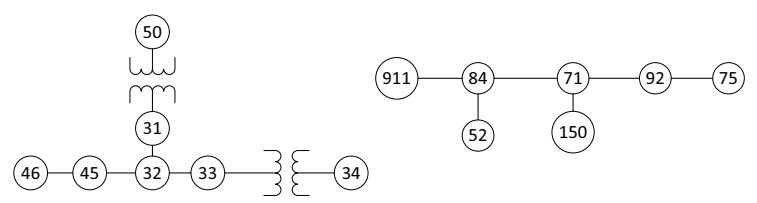

Figure 8: Sub-systems of the IEEE 13-node system

Table 6: Mean squared error in all system measurements for the first sub-system Measurements

\begin{tabular}{c|c|c|c|c|c}
\cline { 2 - 6 } & \multicolumn{3}{|c|}{$(\mathrm{V})$} & \multicolumn{2}{c}{$(\mathrm{A})$} \\
\hline Node & $V_{31}$ & $V_{33}$ & $V_{45}$ & $I_{3132}$ & $I_{3245}$ \\
\hline 45 & 0.000 & 0.006 & 0.014 & 0.001 & 0.000 \\
\hline 46 & 0.000 & 0.072 & 0.142 & 0.190 & 26.774 \\
\hline 45,46 & 0.000 & 0.021 & 0.041 & 0.045 & 6.154 \\
\hline
\end{tabular}

\section{References}

[1] IEEE Recommended Practices and Requirements for Harmonic Control in Electrical Power Systems, IEEE Std 519-1992 (1993) $1-112$.

[2] C. Li, W. Xu, T. Tayjasanant, A "critical impedance"-based method for identifying harmonic sources, IEEE Transactions on Power Delivery 19 (2) (2004) 671-678.

[3] M. Moradloo, M. Tabrizi, H. Karshenas, A new method for identification of main harmonic source based on the superposition and critical impedance methods, in: 40th North American Power Symposium, NAPS '08., 2008, pp. 1-6.

[4] C. Chen, X. Liu, D. Koval, W. Xu, T. Tayjasanant, Critical Impedance Method A New Detecting Harmonic Sources Method in Distribution Systems, IEEE Transactions on Power Delivery 19 (1) (2004) 288-297.

[5] M. Farhoodnea, A. Mohamed, H. Shareef, H. Zayandehroodi, An enhanced method for contribution assessment of utility and customer harmonic distortions in radial and weakly meshed distribution systems, International Journal of Electrical Power \& Energy Systems 43 (1) (2012) 222-229.

[6] J. Mazumdar, G. Venayagamoorthy, R. Harley, Application of neural networks for data modeling of power systems with time varying nonlinear loads, in: IEEE Symposium on Computational Intelligence and Data Mining. CIDM 2007., 2007, pp. 705-711.

[7] P. V. Barbaro, A. Cataliotti, V. Cosentino, S. Nuccio, A Novel Approach Based on Nonactive Power for the Identification of Disturbing Loads in Power Systems, IEEE Transactions on Power Delivery 22 (3) (2007) 1782-1789.

[8] W. Xu, Y. Liu, A method for determining customer and utility harmonic contributions at the point of common coupling, IEEE Transactions on Power Delivery 15 (2) (2000) 804-811.

[9] K. Wilkosz, Single-point measurement localization of prevailing harmonic sources in a power system, in: 11th International Conference on Environment and Electrical Engineering (EEEIC), 2012, pp. 1-6. doi:10.1109/EEEIC.2012.6221387.

[10] H. Liao, Power system harmonic state estimation and observability analysis via sparsity maximization, IEEE Transactions on Power Systems 22 (1) (2007) 15-23. 


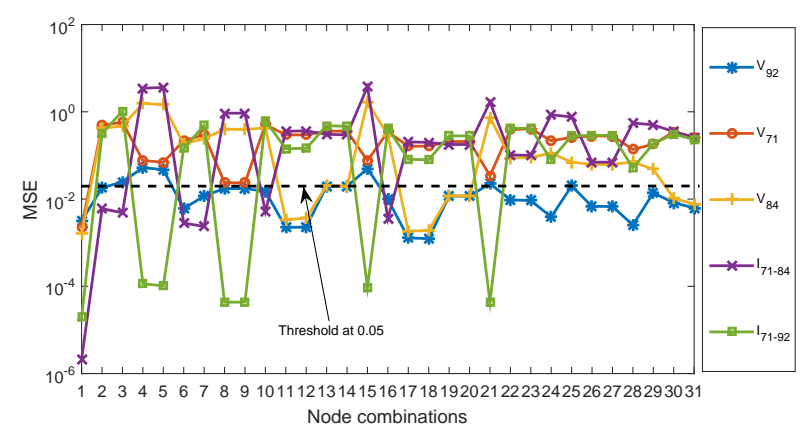

Figure 9: MSE of all combinations for the second sub-system

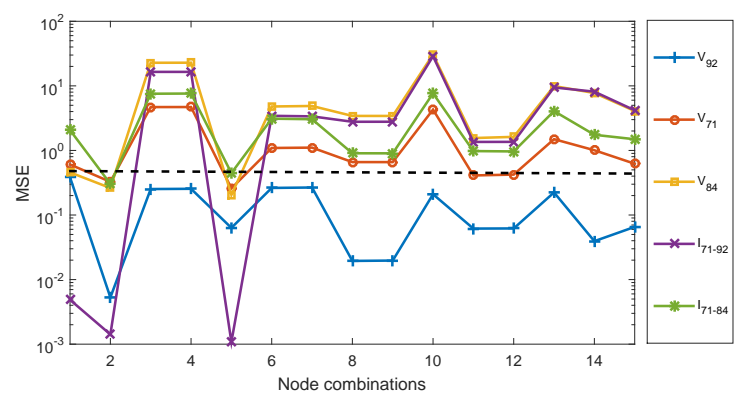

Figure 10: Mean squared error for additional harmonic source at node 75

[11] C. Rakpenthai, S. Uatrongjit, N. Watson, S. Premrudeepreechacharn, On harmonic state estimation of power system with uncertain network parameters, IEEE Transactions on Power Systems 28 (4) (2013) 4829-4838.

[12] H. Beides, G. Heydt, Dynamic state estimation of power system harmonics using Kalman filter methodology, IEEE Transactions on Power Delivery, 6 (4) (1991) 1663-1670.

[13] K. Yu, N. Watson, J. Arrillaga, An Adaptive Kalman Filter for Dynamic Harmonic State Estimation and Harmonic Injection Tracking, IEEE Transactions on Power Delivery 20 (2) (2005) 1577-1584.

[14] A. Kumar, B. Das, J. Sharma, Robust dynamic state estimation of power system harmonics, International Journal of Electrical Power \& Energy Systems 28 (1) (2006) 65-74.

[15] H. Ma, A. Girgis, Identification and tracking of harmonic sources in a power system using a Kalman filter, IEEE Transactions on Power Delivery 11 (3) (1996) 1659-1665.

[16] K. Kennedy, G. Lightbody, R. Yacamini, Power system harmonic analysis using the Kalman filter, 2003 IEEE Power Engineering Society General Meeting 752-757.

[17] A. Medina, R. Cisneros-Magana, Time-domain harmonic state estimation based on the kalman filter poincare map and extrapolation to the limit cycle, IET Generation, Transmission Distribution 6 (12) (2012) 1209-1217.

[18] R. Cisneros-Magaa, A. Medina, J. Segundo-Ramrez, Efficient time domain power quality state estimation using the enhanced numerical differentiation newton type method, International Journal of Electrical Power \& Energy Systems 63 (2014) $414-422$.

[19] H. Mazin, W. Xu, B. Huang, Determining the harmonic impacts of multiple harmonic-producing loads, IEEE Transactions on Power Delivery 26 (2) (2011) 1187-1195. 
[20] D. Saxena, S. Bhaumik, S. Singh, Identification of Multiple Harmonic Sources in Power System using Optimally Placed Voltage Measurement Devices, IEEE Transactions on Industrial Electronics 61 (5) (2014) 2483-2492.

[21] G. D'Antona, C. Muscas, S. Sulis, Localization of nonlinear loads in electric systems through harmonic source estimation, IEEE Transactions on Instrumentation and Measurement 60 (10) (2011) 3423-3430.

[22] W.-M. Lin, C.-H. Lin, K.-P. Tu, C.-H. Wu, Multiple harmonic source detection and equipment identification with cascade correlation network, IEEE Transactions on Power Delivery 20 (3) (2005) 2166-2173.

[23] A. Ujile, Z. Ding, An iterative observer for harmonic estimation in power distribution networks, in: 2014 IEEE PES Transmission and Distribution Conference and Exposition, 2014, pp. 1-5.

[24] W. Chen, J. Yang, L. Guo, S. Li, Disturbance-observer-based control and related methods; an overview, IEEE Transactions on Industrial Electronics 63 (2) (2016) 1083-1095. doi:10.1109/TIE.2015.2478397.

[25] S. Li, J. Yang, W.-H. Chen, X. Chen, Disturbance Observer-Based Control: Methods and Applications, 1st Edition, CRC Press, Inc., Boca Raton, FL, USA, 2014.

[26] K. Yu, N. Watson, An approximate method for transient state estimation, IEEE Transactions on Power Delivery 22 (3) (2007) 1680-1687.

[27] R. Abu-Hashim, R. Burch, G. Chang, M. Grady, E. Gunther, M. Halpin, C. Harziadonin, Y. Liu, M. Marz, T. Ortmeyer, V. Rajagopalan, S. Ranade, P. Ribeiro, T. Sim, W. Xu, Test systems for harmonics modeling and simulation, IEEE Transactions on Power Delivery 14 (2) (1999) 579-587.

[28] G. D'Antona, C. Muscas, P. Pegoraro, S. Sulis, Harmonic source estimation in distribution systems, IEEE Transactions on Instrumentation and Measurement, 60 (10) (2011) 3351-3359.

[29] K. Selvajyothi, P. A. Janakiraman, Extraction of Harmonics Using Composite Observers, IEEE Transactions on Power Delivery 23 (1) (2008) 31-40.

[30] W. Kersting, Radial distribution test feeders, IEEE Transactions on Power Systems 6 (3) (1991) 975-985. 